PROCEEDINGS OF THE

AMERICAN MATHEMATICAL SOCIETY

Volume 127, Number 3, March 1999, Pages 783-789

S 0002-9939(99)04588-8

\title{
POSITIVITY OF POLARIZATIONS OF $n$-POSITIVE MAPS
}

\author{
PIOTR KICIŃSKI
}

(Communicated by Palle E. T. Jorgensen)

\begin{abstract}
It is shown that polarization formulas have explicit matrix representations. This enables us to prove that polarization formulas of $n$-positive maps between $C^{*}$-algebras are coordinatewise positive.
\end{abstract}

\section{INTRODUCTION}

Ando and Choi have characterized completely positive nonlinear maps between $C^{*}$-algebras (cf. [1]). They have proved that such maps can be represented as the sum of completely positive maps which are mixed homogeneous. In general, positive definite maps need not be completely positive. However, as is shown in [3], this is the case for holomorphic maps defined on commutative $W^{*}$-algebras. One way of extending the last mentioned result to commutative $C^{*}$-algebras is to prove that the polarization of any continuous positive definite $k$-homogeneous polynomial is positive definite (this is Question 3 in [3]). In the present paper we show, among other things, that an $n$-polarization of a $\left(2^{n}-1\right)$-positive map (preserving 0 ) between $C^{*}$-algebras is coordinatewise positive. This result is proved with the help of the general matrix representation of polarization formulas for arbitrary maps (preserving 0).

\section{Preliminaries}

In this section we gather all the facts needed for the proof of the main result.

Let $E$ be a $C^{*}$-algebra. By $M_{n}(E)$ we denote the set of $n$-square matrices $a=\left[a_{i j}\right]$, where $a_{i j} \in E$. With the obvious matrix multiplication and the ${ }^{*_{-}}$ operation, $M_{n}(E)$ is a $C^{*}$-algebra.

Lemma 1 ([2, Lem. 3.1]). An element of $M_{n}(E)$ is positive if and only if it is a sum of matrices of the form $\left[a_{i}^{*} a_{j}\right]$ with $a_{1}, \ldots, a_{n} \in E$.

An immediate consequence of Lemma 1 is

Received by the editors June 20, 1997.

1991 Mathematics Subject Classification. Primary 43A35.

Key words and phrases. Completely positive map, polarization of nonlinear map, positive matrix.

(C)1999 American Mathematical Society 
Corollary 2. If $a \geq 0$, then the matrix $A$ of the form

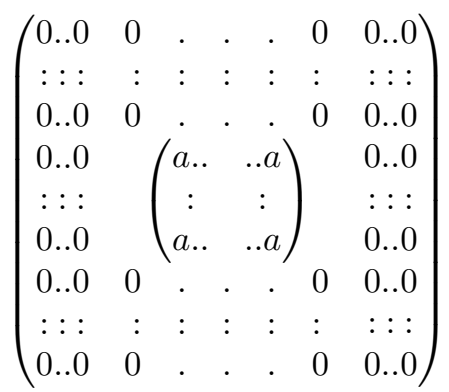

is positive, where the location of the small matrix is such that the main diagonal of the small matrix is contained in the main diagonal of $A$.

Proof. Having established that the location of the small matrix on the main diagonal of $A$ (counting from the left, upper corner of $A$ ) starts from position $m$ and ends on position $n$, then, setting $a_{i}:=a^{1 / 2}$ for $m \leq i \leq n$ and $a_{j}=0$ otherwise, $A$ equals $\left[a_{i}^{*} a_{j}\right]$, so we can apply Lemma 1 .

Corollary 3. If $A \in M_{n}(E)$ is positive, then the matrix $B \in M_{2 n}(E)$ defined by

$$
B=\left(\begin{array}{ll}
A & A \\
A & A
\end{array}\right)
$$

is positive.

Proof. If $A \in M_{n}(E)$ is positive, then by Lemma 1 it is a sum of matrices of the form $\left[a_{i}^{*} a_{j}\right]$. If we then define $b_{i}=b_{n+i}:=a_{i}$ for $1 \leq i \leq n$, then the matrix $B$ is the sum of matrices of the form $\left[b_{k}^{*} b_{l}\right]$, where $1 \leq k, l \leq 2 n$. So by Lemma 1 it is positive.

Lemma 4 ([2, Lem. 3.2]). A matrix $a=\left[a_{i, j}\right] \in M_{n}(E)$ is positive if and only if $\sum_{i, j=1}^{n} x_{i}^{*} a_{i, j} x_{j} \geq 0$ for all $x_{1}, \ldots, x_{n} \in E$.

Definition. Let $E$ and $F$ be $C^{*}$-algebras. Then $\phi: E \longrightarrow F$ is said to be:

(i) n-positive, if for every positive $n$-square matrix $\left[a_{i j}\right]$ with elements in $E$, the matrix $\left[\phi\left(a_{i j}\right)\right]$ with elements in $F$ is also positive.

(ii) completely positive, if it is $n$-positive for every integer $n \geq 1$.

Theorem 5 ([1, Theorem 2]). For any completely positive map $\phi: E \longrightarrow F$ between $C^{*}$-algebras there are completely positive maps $\phi_{m, n}: E \longrightarrow F(m, n=$ $0,1, \ldots)$ such that

$$
\begin{array}{r}
\phi(a)=\sum_{m=0}^{\infty} \sum_{n=0}^{\infty} \phi_{m, n}(a) \quad(a \in E) \quad(\text { norm convergence }), \\
\phi_{m, n}(z a)=z^{m} \bar{z}^{n} \phi_{m, n}(a) \quad(z \in \mathbb{C}, a \in E) .
\end{array}
$$

\section{Homogeneous case}

In this section we prove a result concerning the polarization formula for $k$ homogeneous completely positive polynomials. 
Definition. Let $E$ and $F$ be $C^{*}$-algebras and let $\Phi: E \longrightarrow F$ be an arbitrary map. Then by $\Phi_{n}^{\#}$ we denote the map $\Phi_{n}^{\#}: E^{n} \longrightarrow F$ defined by

$$
\Phi_{n}^{\#}\left(a_{1}, \ldots, a_{n}\right):=\frac{1}{n !} \sum_{i_{1}=0}^{1} \cdots \sum_{i_{n}=0}^{1}(-1)^{n-\left(i_{1}+\cdots+i_{n}\right)} \Phi\left(i_{1} a_{1}+\cdots+i_{n} a_{n}\right) .
$$

Theorem 6 ([1, Theorem 5]). If a completely positive map $\phi: E \longrightarrow F$ is $(m, n)$ mixed homogeneous with $m+n>0$, i.e.

$$
\phi(z a)=z^{m} \bar{z}^{n} \phi(a) \quad(z \in \mathbb{C}, a \in E),
$$

then there is a completely positive map $\Phi: E^{m+n} \longrightarrow F$ such that

$$
\phi(a)=\Phi(a, \ldots, a) \quad(a \in E),
$$

and such that $\Phi\left(a_{1}, \ldots, a_{m+n}\right)$ is multi-linear in $\left(a_{1}, \ldots, a_{m}\right)$ and multi-conjugatelinear in $\left(a_{m+1}, \ldots, a_{m+n}\right)$.

Proposition 7. Let $\Phi: E \longrightarrow F$ be a k-homogeneous completely positive polynomial. If $k \geq n-1$, then $\Phi_{n}^{\#}: E^{n} \rightarrow F$ equals 0 .

Proof. By Theorem 6 there is a completely positive $k$-linear map $\Psi: E^{k} \longrightarrow F$ such that

$$
\Phi(a)=\Psi(a, \ldots, a) .
$$

Now it is sufficient to prove that for all $a_{1}, \ldots, a_{n}$ in $E$ we have

$$
\sum_{i_{1}=0}^{1} \cdots \sum_{i_{n}=0}^{1}(-1)^{n-\left(i_{1}+\cdots+i_{n}\right)} \Psi\left(i_{1} a_{1}+\cdots+i_{n} a_{n}, \ldots, i_{1} a_{1}+\cdots+i_{n} a_{n}\right)=0 .
$$

But, according to the fact that $\Phi$ is $k$-homogeneous, we have

$$
\begin{array}{r}
\sum_{i_{1}=0}^{1} \cdots \sum_{i_{n}=0}^{1}(-1)^{n-\left(i_{1}+\cdots+i_{n}\right)} \Psi\left(\sum_{s_{1}=1}^{n} i_{s_{1}} a_{s_{1}}, \ldots, \sum_{s_{k}=1}^{n} i_{s_{k}} a_{s_{k}}\right)= \\
\sum_{i_{1}=0}^{1} \cdots \sum_{i_{n}=0}^{1}(-1)^{n-\left(i_{1}+\cdots+i_{n}\right)} \sum_{s_{1}=1}^{n} \cdots \sum_{s_{k}=1}^{n} i_{s_{1}} \cdots \cdots i_{s_{k}} \Psi\left(a_{s_{1}}, \ldots, a_{s_{k}}\right)= \\
\sum_{s_{1}=1}^{n} \cdots \sum_{s_{k}=1}^{n}\left(\sum_{i_{1}=0}^{1} \cdots \sum_{i_{n}=0}^{1}(-1)^{n-\left(i_{1}+\cdots+i_{n}\right)} i_{s_{1}} \cdots i_{s_{k}}\right) \Psi\left(a_{s_{1}}, \ldots, a_{s_{k}}\right) .
\end{array}
$$

So it is sufficient to show that for all $s_{1}, \ldots, s_{k} \in\{1, \ldots, n\}$ we have

$$
\sum_{i_{1}=0}^{1} \cdots \sum_{i_{n}=0}^{1}(-1)^{n-\left(i_{1}+\cdots+i_{n}\right)} i_{s_{1}} \cdots i_{s_{k}}=0
$$

But $i_{s_{1}} \cdots i_{s_{k}} \neq 0$ iff $i_{s_{1}}=\cdots=i_{s_{k}}=1$, so if $r$ denotes the number of distinct elements of the set $\left\{s_{1}, \ldots, s_{k}\right\}$ and $\left\{j_{1}, \ldots, j_{n-r}\right\}=\{1, \ldots, n\} \backslash\left\{s_{1}, \ldots, s_{n}\right\}$, then the left hand side of the above equality, after setting $i_{s_{1}}=\cdots=i_{s_{k}}=1$, takes the form

$$
\sum_{i_{j_{1}}=0}^{1} \cdots \sum_{i_{j_{n-r}}=0}^{1}(-1)^{n-r-\left(i_{j_{1}}+\cdots+i_{j_{n-r}}\right)}=\sum_{k_{1}=0}^{1} \cdots \sum_{k_{n-r}=0}^{1}(-1)^{n-r-\left(k_{1}+\cdots+k_{n-r}\right)} .
$$


Hence taking into account that for every integer $p \geq 0$

$$
\sum_{j_{1}=0}^{1} \cdots \sum_{j_{p}=0}^{1}(-1)^{p-\left(j_{1}+\cdots+j_{p}\right)}=\underbrace{(1-1) \cdots \cdots(1-1)}_{p \text {-times }}=0,
$$

we come to the conclusion.

\section{General case}

We begin with a recurrence definition of the sequence of matrices which play a crucial role in the proofs of Theorems 8 and 9 .

Let $E$ and $F$ be $C^{*}$-algebras. Using a recurrence procedure, we define for every $\left(a_{1}, \ldots, a_{n}\right) \in E^{n}$, the $\left(2^{n}-1\right)$-square matrix $A_{n}=\left[a_{i, j}^{n}\right]$. For $n=1$, we set $A_{1}=\left[a_{1}\right]$. If $n \geq 2$, then we put $A_{n}:=D_{n}+C_{n}$, where $D_{n}$ and $C_{n}$ are $\left(2^{n}-1\right)$ square matrices given by

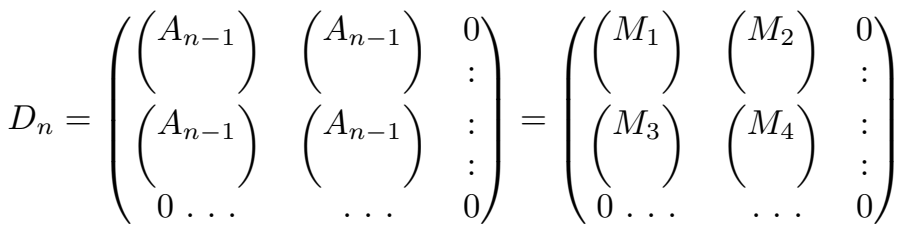

$$
\begin{aligned}
& =\left(\begin{array}{ll}
\left(\begin{array}{c}
M_{1} \\
\end{array}\right) & \left(\begin{array}{c}
M_{2} \\
M
\end{array}\right) \\
\left(\begin{array}{c}
M_{3} \\
\end{array}\right) & \left(\begin{array}{c}
P \\
0 \ldots
\end{array}\right)
\end{array}\right)
\end{aligned}
$$

and

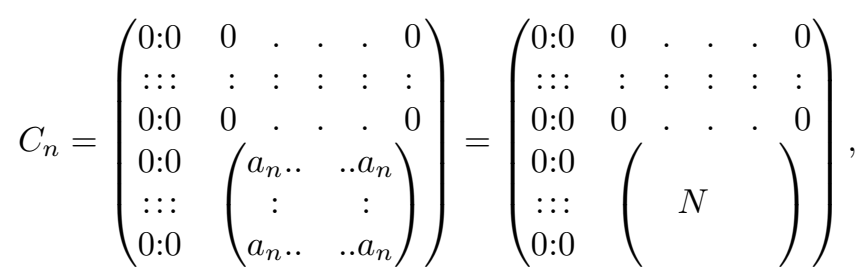

with $M_{1}=M_{2}=M_{3}=M_{4}=A_{n-1} ; N$ and $P$ are $2^{n-1}$ - square matrices. Thus $A_{n}$ is of the form

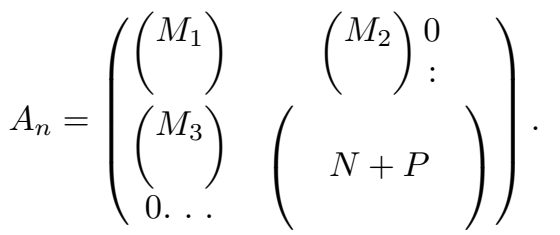

Our main result can be formulated as follows:

Theorem 8. Let $E$ and $F$ be $C^{*}$-algebras and let $\Phi: E \longrightarrow F$ be a map such that $\Phi(0)=0$. Then for every $\left(a_{1}, \ldots, a_{n}\right) \in E^{n}$, we have

$$
n ! \Phi_{n}^{\#}\left(a_{1}, \ldots, a_{n}\right)=\sum_{i, j=1}^{2^{n}-1}(-1)^{i+j} \Phi\left(a_{i, j}^{n}\right) .
$$


Proof. We proceed by induction. Assume that the conclusion holds for integers $1, \ldots, n-1$. Note that in the expression

$$
n ! \Phi_{n}^{\#}\left(a_{1}, \ldots, a_{n}\right)=\sum_{i_{1}=0}^{1} \cdots \sum_{i_{n}=0}^{1}(-1)^{n-\left(i_{1}+\cdots+i_{n}\right)} \Phi\left(i_{1} a_{1}+\cdots+i_{n} a_{n}\right)
$$

we can distinguish summands corresponding to $i_{n}=1$ and to $i_{n}=0$, respectively, i.e.

$$
\begin{array}{r}
\sum_{i_{1}=0}^{1} \cdots \sum_{i_{n-1}=0}^{1}(-1)^{n-\left(i_{1}+\cdots+i_{n-1}+1\right)} \Phi\left(i_{1} a_{1}+\cdots+i_{n-1} a_{n-1}+a_{n}\right) \\
\quad+\sum_{i_{1}=0}^{1} \cdots \sum_{i_{n-1}=0}^{\#}\left(a_{1}, \ldots, a_{n}\right) \\
=\sum_{i_{1}=0}^{1} \cdots \sum_{i_{n-1}=0}^{1}(-1)^{n-\left(i_{1}+\cdots+i_{n-1}+0\right)} \Phi\left(i_{1} a_{1}+\cdots+i_{n-1} a_{n-1}\right) \\
\quad-\sum_{i_{1}=0}^{1} \cdots \sum_{i_{n-1}=0}^{1}(-1)^{n-1-\left(i_{1}+\cdots+i_{n-1}\right)} \Phi\left(i_{1} a_{1}+\cdots+i_{n-1} a_{n-1}\right) \\
=\sum_{i_{1}=0}^{1} \cdots \sum_{i_{n-1}=0}^{1}(-1)^{n-1-\left(i_{1}+\cdots+i_{n-1}\right)} \Phi\left(i_{1} a_{1}+\cdots+i_{n-1} a_{n-1}+a_{n}\right) \\
-(n-1) ! \cdot \Phi_{n-1}^{\#}\left(a_{1}, \ldots, a_{n-1}\right) .
\end{array}
$$

Set $b_{i, j}^{n}=\Phi\left(a_{i, j}^{n}\right)$ and $B_{n}=\left[b_{i, j}^{n}\right]$. Then, by the assumption $\Phi(0)=0$, we have

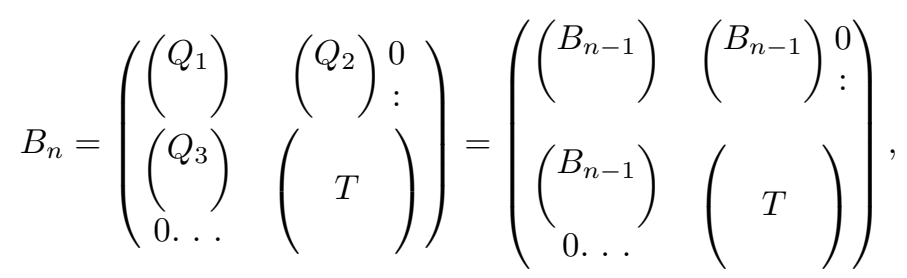

where $Q_{1}=Q_{2}=Q_{3}=B_{n-1}$. Set $I_{1}=\left\{(i, j): 1 \leq i, j \leq 2^{n-1}-1\right\}, I_{2}=$ $I_{1}+\left(2^{n-1}-1,0\right), I_{3}=I_{1}+\left(0,2^{n-1}-1\right), I_{4}=I_{1}+\left(2^{n-1}-1,2^{n-1}-1\right)$ and $J=\left\{(i, j): 2^{n-1} \leq i, j \leq 2^{n}-1\right\}$. If $(i, j) \in I_{1}$, then $b_{i, j}^{n}=b_{i+2^{n-1}-1, j}^{n}=$ $b_{i, j+2^{n-1}-1}^{n}=b_{i, j}^{n-1}$, so

$$
\sum_{(i, j) \in I_{1} \cup I_{2} \cup I_{3}}(-1)^{i+j} b_{i, j}^{n}=-\sum_{(i, j) \in I_{1}}(-1)^{i+j} b_{i, j}^{n-1} .
$$

Consequently, by the induction assumption, we have

$$
\sum_{(i, j) \in I_{1} \cup I_{2} \cup I_{3}}(-1)^{i+j} b_{i, j}^{n}=-(n-1) ! \cdot \Phi_{n-1}^{\#}\left(a_{1}, \ldots, a_{n-1}\right) .
$$

To end the proof it is sufficient to show that $\sum_{(i, j) \in J}(-1)^{i+j} b_{i, j}^{n}$ is equal to the first term appearing on the right hand side of (2). Set $\Psi(x)=\Phi\left(x+a_{n}\right)-\Phi\left(a_{n}\right)$ 
for $x \in E$ and $\tilde{b}_{i, j}^{n-1}=\Psi\left(a_{i, j}^{n-1}\right)$ for $(i, j) \in I_{1}$. Then we have

$$
\tilde{b}_{i, j}^{n-1}=b_{i+2^{n-1}-1, j+2^{n-1}-1}^{n}-\Phi\left(a_{n}\right), \quad(i, j) \in I_{1} .
$$

It follows from (1), the induction assumption applied to $\Psi$ and (3) that

$$
\begin{gathered}
\sum_{i_{1}=0}^{1} \cdots \sum_{i_{n-1}=0}^{1}(-1)^{n-1-\left(i_{1}+\cdots+i_{n-1}\right)} \Phi\left(i_{1} a_{1}+\cdots+i_{n-1} a_{n-1}+a_{n}\right) \\
=\sum_{i_{1}=0}^{1} \cdots \sum_{i_{n-1}=0}^{1}(-1)^{n-1-\left(i_{1}+\cdots+i_{n-1}\right)}\left(\Phi\left(i_{1} a_{1}+\cdots+i_{n-1} a_{n-1}+a_{n}\right)-\Phi\left(a_{n}\right)\right) \\
\quad+\sum_{i_{1}=0}^{1} \cdots \sum_{i_{n-1}=0}^{1}(-1)^{n-1-\left(i_{1}+\cdots+i_{n-1}\right)} \Phi\left(a_{n}\right) \\
=\sum_{i_{1}=0}^{1} \cdots \sum_{i_{n-1}=0}^{1}(-1)^{n-1-\left(i_{1}+\cdots+i_{n-1}\right)} \Psi\left(i_{1} a_{1}+\cdots+i_{n-1} a_{n-1}\right) \\
=(n-1) ! \cdot \Psi_{n-1}^{\#}\left(a_{1}, \ldots, a_{n-1}\right)=\sum_{(i, j) \in I_{1}}(-1)^{i+j} \tilde{b}_{i, j}^{n-1} \\
=\sum_{(i, j) \in I_{4}}(-1)^{i+j} b_{i, j}^{n}-\sum_{(i, j) \in I_{4}}(-1)^{i+j} \Phi\left(a_{n}\right) \\
=\sum_{(i, j) \in I_{4}}(-1)^{i+j} b_{i, j}^{n}-\Phi\left(a_{n}\right) .
\end{gathered}
$$

Since

$$
\sum_{(i, j) \in J \backslash I_{4}}(-1)^{i+j} b_{i, j}^{n}=\left(\sum_{k=2^{n-1}}^{2^{n}-1}(-1)^{k+2^{n}-1}+\sum_{k=2^{n-1}}^{2^{n}-2}(-1)^{k+2^{n}-1}\right) \Phi\left(a_{n}\right)=-\Phi\left(a_{n}\right),
$$

the proof is complete.

Theorem 8 provides us with a handy tool. Here we have one possible application.

Theorem 9. Let $E$ and $F$ be $C^{*}$-algebras. Assume that a map $\Phi: E \longrightarrow F$ is $\left(2^{n}-1\right)$-positive and that $\Phi(0)=0$. Then for all $a_{1}, \ldots, a_{n} \geq 0$ in $E$ we have $\Phi_{n}^{\#}\left(a_{1}, \ldots, a_{n}\right) \geq 0$.

Proof. We preserve the notation from Theorem 8. By induction we show that all the matrices $A_{n}$ are positive for $a_{1}, \ldots, a_{n} \geq 0$.

The case $n=1$ is trivial.

Assume that $A_{n-1}$ is positive for $a_{1}, \ldots, a_{n-1} \geq 0(n \geq 2)$. Take $a_{n} \geq 0$. Then, by Corollaries 2 and 3, the matrices $C_{n}$ and $D_{n}$ are positive. Hence so is their sum $A_{n}=C_{n}+D_{n}$.

Since $A_{n}$ is positive and $\Phi$ is $\left(2^{n}-1\right)$-positive, the matrix $B_{n}=\left[\Phi\left(a_{i j}\right)\right]$ is positive as well. Set $X_{n}=[x,-x, x,-x, \ldots,-x, x] \in E^{2^{n}-1}$. Then, by Theorem 8 and Lemma 4, we have

$$
x\left(n ! \Phi_{n}^{\#}\left(a_{1}, \ldots, a_{n}\right)\right) x^{*}=x\left(\sum_{i, j=1}^{2^{n}-1}(-1)^{i+j} \Phi\left(a_{i, j}^{n}\right)\right) x^{*}=X_{n} B_{n}\left(X_{n}^{T}\right)^{*} \geq 0, \quad x \in F,
$$

which, according to Lemma 4 , yields $\Phi_{n}^{\#}\left(a_{1}, \ldots, a_{n}\right) \geq 0$. 
Corollary 10. If $\Phi$ is completely positive and $\Phi(0)=0$, then $\Phi_{n}^{\#}\left(a_{1}, \ldots, a_{n}\right) \geq 0$ for every integer $n \geq 0$ and for all $a_{1}, \ldots, a_{n} \geq 0$.

\section{REFERENCES}

1. Ando, T., Choi, M.-D., Non-Linear Completely Positive Maps, Aspects of Positivity in Functional Analysis, 1986. MR 88a:46059

2. Takesaki, Masamichi, Theory of Operator Algebras I, Springer-Verlag, New York, Heidelberg, Berlin, 1979. MR 81e:46038

3. Stochel, Jan, Decomposition and Disintegration of Positive Definite Kernels on Convex *Semigroups, Annales Polonici Mathematici 56 (1992). MR 93g:43003

4. Ando T., Inequalities for permanents, Hokkaido Math. J. 10 (1981). MR 83i:15010

5. Schoenberg, I.J., Positive Definite Functions on Spheres, Duke Math. J. 9 (1942), 96-108. MR 3:232c

Instytut Matematyki UJ, Ul.Reymonta 4, PL-30059, Krakøw, Poland

E-mail address: kicinski@eta.im.uj.edu.pl 